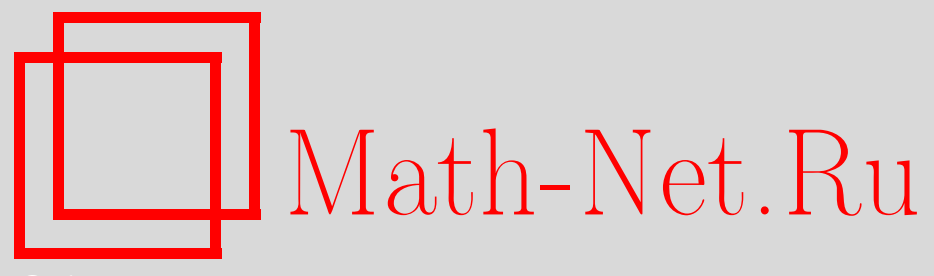

А. М. Степин, С. В. Тихонов, Замечания об изожесткости, централизаторах и спектральной эквивалентности в эргодической теории, Матем. заметки, 2007, том 81, выпуск 2, 314-316

DOI: https://doi.org/10.4213/mzm3558

Использование Общероссийского математического портала Math-Net.Ru подразумевает, что вы прочитали и согласны с пользовательским соглашением http://www.mathnet.ru/rus/agreement

Параметры загрузки:

IP: 52.23 .180 .231

26 апреля 2023 г., 15:57:41

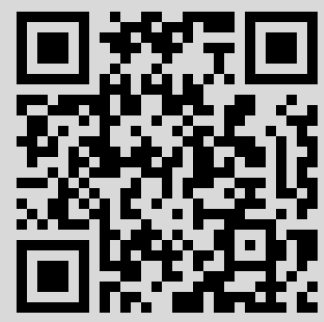




\section{Замечания об изожесткости, централизаторах и спектральной эквивалентности в эргодической теории}

\section{А. М. Степин, С. В. Тихонов}

Предмет этой заметки - свойства обратимых преобразований $T: M \rightarrow M$, сохраняющих конечную меру $\mu$ на $M$; такие преобразования называют также автоморфизмами пространства с мерой $(M, \Sigma, \mu)$. О некоторых из асимптотических или алгебраических свойств этих преобразований иногда полезно говорить как о типичных или нетипичных. Для придания одного из возможных точных значений выражению "типичное свойство" используется слабая топология в группе $\mathscr{A}$ всех автоморфизмов пространства $(M, \Sigma, \mu)$. Эта топология задается системой окрестностей вида

$$
\mathscr{O}(T ; A, \varepsilon)=\left\{S \in \mathscr{A} \mid \mu(T A \triangle S A)+\mu\left(T^{-1} A \Delta S^{-1} A\right)<\varepsilon\right\},
$$

где $A \in \Sigma, \varepsilon>0$ и $\Delta-$ операция симметрической разности. Топологическая группа $\mathscr{A}$ полна относительно слабой топологии, и это позволяет говорить о массивных подмножествах в $\mathscr{A}$, т.е. счетных пересечениях всюду плотных открытых множеств. То или иное свойство преобразований сохраняющих меру типично, если оно выполнено для массивного множества элементов из $\mathscr{A}$.

1. Пусть $R$ - некоторое бинарное отношение автоморфизмов, согласованное с изоморфизмом преобразований; последний это синоним сопряженности в $\mathscr{A}$. Через $f_{R}(T)$ обозначим наибольшую мощность семейств $\left\{T_{\alpha}\right\} \subset \mathscr{A}$, элементы которых попарно неизоморфны и для всех $\alpha$ имеем $T_{\alpha} \underset{R}{\sim} T$. Таким образом, $f_{R}(T)$ есть кардинальнозначный инвариант преобразования $T$. В качестве $R$ здесь будут рассмотрены следующие соотношения, первое из которых не является отношением эквивалентности:

1. Sq: имеем $T_{1}^{2}=T_{2}$;

2. $\mathrm{SE}: T_{1}$ и $T_{2}$ спектрально эквивалентны и имеют нулевую энтропию;

3. IC: централизаторы преобразований $T_{1}$ и $T_{2}$ изоморфны;

4. IR: для любой последовательности $\left\{k_{i}\right\} \subset \mathbb{N}$, имеем $T_{1}^{k_{i}} \rightarrow I \Leftrightarrow T_{2}^{k_{i}} \rightarrow I$ при $i \rightarrow \infty$.

Последнее отношение естественно назвать изожесткостью.

$\mathrm{K}$ интересным задачам в эргодической теории приводят вопросы о том, какие значения может принимать инвариант $f_{R}(T)$, каковы его максимальные значения и какие значения типичны. Хотя ответы на первый вопрос могут быть весьма интересны, мы не будем останавливаться на нем в этой заметке.

$\mathrm{C}$ помощью инварианта $f_{R}$ можно переформулировать некоторые известные результаты эргодической теории. Например, теорема Кинга о корнях утверждает, что в типичном случае $f_{\mathrm{Sq}} \geqslant 1$, а результат, полученный в [1], [2] максимально уточняет это неравенство: для типичного $T$, имеем $f_{\mathrm{Sq}}=\complement$ (здесь $\complement$ - мощность континуума). В пп. 3 и 4 будут рассмотрены вопрос Гудзона о возможных (и типичных) значениях $f_{\mathrm{SE}}$ и вопрос о числе неизоморфных изожестких преобразований, обсуждавшийся на конференции в Торуни в 2001 г.

2. Здесь сформулированы факты, которые будут использованы в пп. 3 и 4 . Одним из унифицирующих способов построения преобразований с "необычными" свойствами в эргодической теории является машина контрпримеров Рудольфа. Искомые преобразования она представляет в виде композиций бесконечных декартовых произведений некоторого преобразования и перестановок сомножителей. Еще одна машина контрпримеров, основанная на свойстве дизъюнктности сверточных степеней максимального спектрального 
типа, была предложена дель Юнко и Леманчиком [3]; в ней используются декартовы произведения степеней типичного преобразования и не используются упомянутые выше перестановки.

Централизатор автоморфизма $T$ обозначим через

$$
C(T)=\{S \in \mathscr{A} \mid S T=T S\} .
$$

Известно, что в типичном случае $C(T)$ есть слабое замыкание степеней преобразования $T$.

Для множества $J \subset \mathbb{Z}$ через $\bigotimes_{j \in J} T^{j}$ обозначим декартово произведение преобразований $\left\{T^{j}\right\}_{j \in J}$.

Теорема 1 (см. [3]). Существует массивное множество преобразований $\mathscr{G}$ такое, что для $T \in \mathscr{G}$ и множества $J \subset \mathbb{Z} \backslash\{0\}$ имеем

$$
C\left(\bigotimes_{j \in J} T^{j}\right)=C(T) \otimes C(T) \otimes \cdots
$$

Кроме того, для различных $J$ произведения $\bigotimes_{j \in J} T^{j}$ неизоморфны.

Вот еще два утверждения, которые будут нам нужны (см. [1], [4]).

Теорема 2. Существует массивное множество $\mathscr{Q}$ преобразований, имеющих континуум попарно неизоморфных корней.

ПреДЛОЖЕНИЕ. Объединение множеств корней из типичных преобразований содержит массивное множество.

3. О максимальных значениях инвариантов $f_{R}$. Как известно, сохраняющее меру преобразование $T$ эквивалентно своему обратному $T^{-1}$ и имеет одинаковую с ним энтропию. Таким образом, классические инварианты эргодической теории не позволяют различить $T$ и $T^{-1}$. Вместе с тем, пример Анзаи [5] говорит о том, что они не всегда изоморфны. Через двадцать лет Оселедец [6] посторил автоморфизм $T$ с простым непрерывным спектром, не изоморфный своему обратному; иначе говоря, для этого преобразования $T$, имеем $f_{\mathrm{SE}}(T) \geqslant 2$. Десятью годами позже дель Юнко показал, что эта оценка верна в типичном случае.

Рассмотрим семейство преобразований

$$
\mathscr{P}=\left\{\bigotimes_{j \in J} S^{j}\right\}_{J \subset \mathbb{Z} \backslash\{0\}},
$$

где для каждого $n \in \mathbb{N}$ либо $n \in J$, либо $-n \in J$.

Потребуем, чтобы преобразование $S$ принадлежало $\mathscr{G}$ и имело нулевую энтропию. Тогда все степени $S$ и их декартовы произведения также имеют нулевую энтропию. Все такие произведения не изоморфны между собой по теореме 1. С другой стороны, нетрудно проверить, что максимальные спектральные типы всех этих преобразований совпадают. Таким образом, для типичного $S$ имеем $f_{\mathrm{SE}}\left(\bigotimes_{j \in \mathbb{N}} S^{j}\right)=$ С. Примеры преобразований с таким значением характеристики $f_{\mathrm{SE}}$ были построены Агеевым. В отличие от его примера, в нашей конструкции спектр может быть сделан простым.

Поскольку, централизаторы всех элементов из $\mathscr{P}$ суть декартовы произведения централизаторов $S$ и, стало быть, совпадают, то

$$
f_{\mathrm{IC}}\left(\bigotimes_{j \in \mathbb{N}} S^{j}\right)=\complement .
$$

Далее, если для некоторой последовательности $\left\{k_{i}\right\} \subset \mathbb{Z}$ и преобразования $T \in \mathscr{P}$ имеем $T^{k_{i}} \rightarrow I$, то сходимость есть и по каждой (декартовой) координате; в частности, $S^{k_{i}} \rightarrow I$. Поскольку в $\mathscr{A}$ непрерывна операция умножения, имеем $\left(S^{n}\right)^{k_{i}} \rightarrow I$ и 
для всех $J \subset \mathbb{Z} \backslash\{0\}$, последовательность $\left(\bigotimes_{j \in J} S^{j}\right)^{k_{i}}$ сходится к тождественному преобразованию при $i \rightarrow \infty$. Таким образом, $S$ и $\left(\bigotimes_{j \in J} S^{j}\right)^{k_{i}}-$ изожесткие преобразования и, поскольку изожесткость есть отношение эквивалентности, то семейство преобразований $\left\{\bigotimes_{j \in J} S^{j}\right\}_{J \subset \mathbb{Z} \backslash\{0\}}$ состоит из попарно изожестких преобразований. Кроме того, они неизоморфны по теореме 1 . Отсюда следует, что $f_{\mathrm{IR}}(S)=\complement$.

4. О типичных значениях инвариантов $f_{R}$. Отыскание таких значений может оказаться непростым делом. В рассматриваемых здесь случаях проще всего дело обстоит с характеристикой $f_{\mathrm{IR}}$, поскольку для $T \in \mathscr{G}$ (см. теорему 1$)$ имеем $f_{\mathrm{IR}}(T)=\complement$.

Теорема. Централизаторы всех корней из типичного преобразования совпадают. Как следствие, для типичного $T$ имеем $f_{\mathrm{IC}}(T)=\complement$.

ДокАЗАтельство. Действительно, пусть $T$ коммутирует лишь со слабым замыканием своих степеней, а $V$ и $W$ - два корня из $T$. Если $S$ коммутирует с $V$, то коммутирует и с $T$. Тогда $S$ представляется как слабый предел некоторой последовательности степеней $T$. В этом случае $W$ коммутирует с $S$, поскольку коммутирует с элементами сходящейся к $S$ последовательности. Таким образом, $C(V) \subset C(W)$ и, следовательно, $C(V)=C(W)$.

По теореме 2 типичное преобразование имеет несчетное (фактически континуальное) семейство попарно неизоморфных корней. Для каждого из них инвариант $f_{\mathrm{IC}}$ принимает значение С. Наконец, по предложению из п. 2 множество таких корней массивно.

\section{СПИСОК ЦИТИРОВАННОЙ ЛИТЕРАТУРЫ}

[1] А. М. Степин, А. М. Еременко, Докл. РАН, 394:6 (2004), 739-742. [2] О. Н. Агеев, УMH, 58:1 (2003), 177-178. [3] M. Lemanczyk, A. del Junco, Proc. Amer. Math. Soc., 115:3 (1992), 725-736. [4] С. В. Тихонов, Докл. РАН, 391:1 (2003), 26-28. [5] H. Anzai, Osaka J. Math., 3:1 (1951), 83-99. [6] В. И. Оселедец, Функи. анализ и его прилож., 5:3 (1971), $75-79$.

А. М. Степин, С. В. Тихонов

Поступило

Московский государственный

27.06.2006

университет им. М. В. Ломоносова 